\section{P418 UPTAKE OF HIV PRE-EXPOSURE PROPHYLAXIS (PREP) IN WESTERN NIGERIA: ARE HEALTHCARE PROVIDERS PREPARED FOR THE KEY POPULATIONS?}

${ }^{1}$ Ibiwumi Usman*, '2Saheed Usman. 'Kids and Teens Resource Centre, Akure, Nigeria; ${ }^{2}$ APIN Public Health Intiatives, Abuja, Nigeria

\subsection{6/sextrans-2019-sti.504}

Background HIV pre-exposure prophylaxis (PrEP) can decrease HIV incidence among several high-risk populations. In order to successfully implement PrEP, healthcare providers will need to have knowledge about counselling, monitoring and drug adherence. This study was carried out to determine the awareness, practice and preparedness of healthcare professionals to prescribe PrEP in clinical settings especially to key populations in our communities and identify the factors associated with or encouraging its prescription.

Methods This cross-sectional study was carried out in randomly selected primary, secondary and tertiary level hospitals in Western Nigeria. The target population were physicians and nurses largely involved in the antiretroviral clinics in the hospitals. Data was collected by trained volunteers and supervised by appointed supervisors by a face-to-face interview. All data were statistically analysed, using Statistical Package for the Social Sciences (SPSS) and statistical test of significance was performed with Chi-Square test.

Results A total of 256 consenting respondents participated in the study with a mean age \pm SD of $38.52 \pm 9.29$ years. A total of $89.8 \%$ of the respondents have heard about PrEP, with $54.3 \%$ of them aware of both oral and topical PrEP while only $4.3 \%$ have ever prescribed PrEP. The main factor associated with PrEP prescription was work experience $\left(\chi^{2}=\right.$ 20.815 , df $=1, \mathrm{p}=0.001)$. Work experience has lower association with PrEP prescription (OR: 0.88, 95\% CI: 0.82$0.95)$.

Conclusion Healthcare professionals in public hospitals in Nigeria are PrEP aware and willing to prescribe, but few have actually ever done the prescription. Regular supply of drugs for pre-exposure prophylaxis purpose and addressing the potential safety issues and medication-related adverse effects will help aid the PrEP implementation effort nationwide especially with focus on the key populations of mem having sex with (MSM) who are in a hostile environment in our own neighbourhood.

Disclosure No significant relationships.

\section{P419 PATTERNS OF GROUP SEX ACTIVITY AMONG GAY AND BISEXUAL MEN IN MELBOURNE AND SYDNEY IN AUSTRALIA, 2013-2018}

${ }^{1}$ Eric Chow* ${ }^{2}$ Toby Lea, ${ }^{1}$ Christopher Fairley, ${ }^{2}$ Limin Mao, ${ }^{2}$ Timothy Broady, ${ }^{3}$ Benjamin Bavinton, ${ }^{3}$ Garrett Prestage, ${ }^{2}$ Martin Holt. ${ }^{1}$ Alfred Health, Melbourne Sexual Health Centre, Carlton, Australia; ${ }^{2}$ UNSW Sydney, Centre for Social Research in Health, Sydney, Australia; ${ }^{3}$ UNSW Sydney, The Kirby Institute, Sydney, Australia

\subsection{6/sextrans-2019-sti.505}

Background This study analysed trends in group sex among gay and bisexual men (GBM) and the factors associated with group sex. It is important to understand GBM who engage in group sex as they may be at risk of and require more intensive screening for sexually transmitted infections (STI).

Methods The Gay Community Periodic Surveys are annual behavioural surveys of GBM. We analysed data collected from
Melbourne and Sydney during 2013-2018. Participants were eligible if they were $\geq 16$ years, male, and reported sex with men in the last 5 years. We calculated trends in group sex (sex involving at least two other men) in the last 6 months and used multivariable logistic regression to identify factors associated with it.

Results Among 31,341 GBM, the proportion engaging in group sex increased from $30.9 \%$ in 2013 to $36.8 \%$ in 2018 $\left(\mathrm{p}_{\text {trend }}<0.001\right)$. Group sex was associated with having $\geq 10$ male partners in the last 6 months (aOR: 3.63; 95\% CI: 3.38-3.90), using Viagra (aOR: 1.40; 95\% CI: 1.29-1.52) and testing for HIV/STI in the last 12 months (aOR: 1.36; 95\% CI: 1.25-1.47). Compared with HIV-negative GBM who did not use PrEP $(n=23,003)$, HIV-negative GBM who used PrEP ( $n=2,299$; aOR: 1.57; 95\% CI: 1.39-1.77) and HIV-positive GBM ( $n=2,701$; aOR: 1.15; 95\% CI: 1.03-1.28) were more likely to report group sex. GBM who met male partners at private sex parties (aOR: 5.03; 95\% CI: 4.30-5.89) and saunas (aOR: 2.34; 95\% CI: 2.17-2.51) had the highest odds of engaging in group sex.

Conclusion Group sex has become more common among GBM in Melbourne and Sydney over the last 6 years. While group sex is concentrated among PrEP users and HIV-positive GBM, there remains a large group of non-PrEP-users who engaged in group sex and would benefit from regular STI screening (and potentially the offer of PrEP).

Disclosure No significant relationships.

\section{P420 UNDERSTANDING PREP SERVICE DELIVERY PREFERENCES AMONG BLACK WOMEN IN URBAN AND RURAL COUNTIES IN THE US DEEP SOUTH}

${ }^{1}$ Latesha Elopre*, ${ }^{1}$ Chastity Mcdavid, ${ }^{1}$ Bernadette Johnson, ${ }^{2}$ Bretia Gordon, ${ }^{3}$ Barbara Van Der Pol, ${ }^{4}$ Jeanne Marrazzo, 'Michael Mugavero. 'University of Alabama at Birmingham, Medicine, Birmingham, USA; ${ }^{2}$ Montgomery Advocacy and Outreach, Montgomery, USA; ${ }^{3}$ University of Alabama at Birmingham, Medicine/Infectious Diseases, Birmingham, USA Minor Outlying Islands; ${ }^{4}$ University of Alabama, USA

\subsection{6/sextrans-2019-sti.506}

Background In the US, women account for approximately $20 \%$ of all new HIV infections, among which two-thirds are Black women. These health disparities are most pronounced in the Deep South. We conducted focus groups in urban and rural settings in Alabama to understand attitudes and preferences for PrEP service delivery among Black women.

Methods Our group members consisted of HIV-negative women between the ages of 16-65. Items and analyses were grounded in a conceptual framework using constructs from two behavioral models. Two coders independently conducted inductive and deductive thematic coding to determine major themes.

Results 25 rural (median age 44, 48\% income $<\$ 25,000$, $36 \%$ Bachelor's degree) and 22 urban residents (median age $35,14 \%$ income $<\$ 25,000,54 \%$ Bachelor's degree) were enrolled. Common themes identified among both groups of women were lack of awareness regarding PrEP and openness to utilization of PrEP if self-perceived risk for HIV was high. Among rural women, many expressed more overall distrust in knowing their partners risk of HIV infection as well as fear of potential disclosure if accessing PrEP within their community. Rural women also reported more concerns about stigma related to PrEP and structural barriers limiting access to service. Many rural women felt churches could be avenues to 